\title{
A protocol used to manage maxillary hypoplasia in cleft lip and palate patients
}

\author{
Ahmed Alyamani*, Sondos Abuzinada* \\ Oral and Maxillofacial Surgery Department, King Abdulaziz University, Jeddah, Saudi Arabia \\ Email: *sondoz@hotmail.com
}

Received 8 February 2012; revised 2 March 2012; accepted 15 March 2012

\begin{abstract}
Objectives: We report our experience and the protocol we used in managing maxillary hypoplasia in cleft lip and palate patients. Patients and methods: 14 adult cleft lip and palate patients with maxillary hypoplasia were evaluated clinically. Dental models and radiographs including (lateral cephalograms and orthopantographs) were obtained at the initial visit and upon completion of the presurgical orthodontic treatment. Patients with occlusal discrepancies larger than $6 \mathrm{~mm}$ and severe palatal scaring underwent Distraction osteogenesis (DO) to advance the maxilla. Patients with an occlusal discrepancy of $6 \mathbf{~ m m}$ or less, underwent traditional orthognathic surgery including le fort I advancement and Bilateral sagittal split osteotomy (BSSO) to seat the mandible in occlusion. Results: Five patients underwent orthognathic surgery. Two of them underwent double jaw surgery. Three underwent single jaw conventional le fort $l$ advancement. Four patients required bone grafting to repair the residual alveolar defect and to augment the midface deficiency. Nine patients with severe maxillary hypoplasia underwent maxillary advancement using distraction osteogenesis. Conclusion: Patients with a severe maxillary hypoplasia of $6 \mathrm{~mm}$ or more and excessive palatal scaring are successfully treated with DO. Conventional le fort $I$ is reserved for patients with less severe maxillary hypoplasia. Both techniques gave promising results providing having followed the proper selection criteria.
\end{abstract}

Keywords: Cleft Lip and Palate; Hypoplasia; Maxilla; Midface; Class III; RED; Distraction Osteogenesis

\section{INTRODUCTION}

Cleft lip and palate patients are borne with a challenging deformity that requires multiple surgical interventions in

\footnotetext{
*Corresponding authors.
}

order to reach functional and esthetic harmony. Unfortunately some of these surgical procedures carry a negative impact along with the positive effect. During infancy and early childhood, surgical repair of the cleft lip and palate is usually done to improve facial appearance and function. However, these surgical interventions have an unpleasant effect on maxillary growth and the child grows into a skeletal class III due to maxillary hypoplasia. Cleft palate repair is usually performed at approximately 9 to 18 months of age for speech development.

The hypoplastic maxilla in cleft patients can be treated using conventional le Fort I advancement with or without bone grafting. However, the surgical advancement in some cases with severe palatal scaring is not an easy task and bares the problem of relapse [1]. On the bright side of the spectrum Distraction osteogenesis (DO) played a huge role in managing midface hypoplasia (DO) was first introduced to the mandible by McCarthy et al. [2], then to the maxilla of cleft lip and palate patients by Polley and Figueroa [3]. This gave very good results in treating the hypoplastic maxilla. Many surgeons applied this valuable technique on cleft lip and palate patients and reported the effectiveness of midface DO [4].

We report our experience with 14 cleft lip and palate patients. They all presented with maxillary hypoplasia associated with a class III malocclusion. They underwent surgical correction using either conventional le Fort I advancement or maxillary DO according to the severity of the condition and the amount of palatal scaring along with other factors. We report our protocol in management of such cases.

\section{PATIENTS AND METHODS}

14 cleft lip and palate patients were referred to the clinics of oral and maxillofacial surgery at king Abdulaziz university hospital between 2005 and 2010. They all presented with a midface hypoplasia and class III skeletal malocclusion. Eight males and six females Their ages ranged between 14 - 26 years.

All of them had undergone surgical repair of their cleft 
lip and palate during their first two years of life. Complete records were obtained including orthopantographs (OPG), lateral cephalometric radiographs and dental models. A treatment plan was proposed according to the severity of the malocclusion, the supporting soft tissue and bony structures. Patients were then referred to the orthodontics department in order to begin orthodontic treatment in preparation for either orthognathic surgery or maxillary advancement using distraction osteogenesis.

After completing the presurgical orthodontic phase patients were referred back to the Oral and maxillofacial surgery department for surgical intervention. All Patients with a velopharyngeal flap were intubated successfully using fiberoptic intubation and the flap was left intact. Patients with occlusal discrepancies larger than $6 \mathrm{~mm}$ and severe palatal scaring underwent Distraction osteogenesis to advance the maxilla. A le Fort I osteotomy was performed and the maxilla was down fractured fixed to the RED using $2 \mathrm{~mm}$ plates and screws. In situations where the maxilla split into a two pieces with the down fracture force, a $2 \mathrm{~mm}$ plate was placed to split the two segments in the anterior maxillary region in addition to an occlusal splint that was prepared preoperatively. After a 7 day latency period the distractor was activated at a rate of $1 \mathrm{~mm}$ per day in 2 rhythms. After completing a three month consolidation period the distractor was removed. In patients with a missing premaxilla due to previous surgical removal during infancy at another center, we grafted the defect using anterior iliac bone graft dur- ing distractor removal. On the other hand ,patients with an occlusal discrepancy of $6 \mathrm{~mm}$ or less with less severe palatal scaring, underwent traditional orthognathic surgery which included le Fort I advancement and Bilateral sagittal split osteotomy (BSSO) to seat the mandible in occlusion without any setback. Any remaining alveolar bone defects in the cleft site were grafted using anterior iliac bone graft (Table 1). Post operative lateral cephalograms and OPG were obtained All patients were referred back to the orthodontics department in order to resumed their treatment and were followed up every 3 months for an average range of 18 months .

\section{RESULTS}

Five patients underwent orthognathic surgery. Two of them underwent double jaw surgery (including le Fort I advancement and BSSO to seat the mandible in occlusion) (Figure 1). Three underwent single jaw conventional le fort 1 advancement. Four patients required bone grafting to repair the residual alveolar defect and to augment the midface deficiency.

Nine patients with severe maxillary hypoplasia underwent maxillary advancement using distraction osteogenesis (Figures 2 and 3). An External rigid distractor (RED) was used in 8 patients and an internal distractor was used in one patient. The average distraction distance was 12 $\mathrm{mm}$. Four patients developed an anterior open bite during the distraction phase. However this was corrected by

Table 1. List of patients with cleft lip and palate treatment methods.

\begin{tabular}{|c|c|c|c|c|c|}
\hline Patient & Cleft type & Premaxilla & Descrepency & Surgical treatment & Follow up \\
\hline 1 & $\mathrm{U}$ & Intact & $6 \mathrm{~mm}$ & Le Fort 1 advancement BSSO/Iliac bone graft & $5 \mathrm{yr}$ \\
\hline 3 & $\mathrm{~B}$ & Intact & $5 \mathrm{~mm}$ & Le Fort 1 advancemnt and iliac bone graft & $3 \mathrm{yr}$ \\
\hline 4 & $\mathrm{U}$ & Intact & $5 \mathrm{~mm}$ & Le Fort 1 advancemnt & $3 \mathrm{yr}$ \\
\hline 5 & $\mathrm{U}$ & Intact & $6 \mathrm{~mm}$ & Le Fort I advancement & $4 \mathrm{yr}$ \\
\hline 6 & $\mathrm{U}$ & Intact & $9 \mathrm{~mm}$ & RED then face mask & $3 \mathrm{yr}$ \\
\hline 7 & $\mathrm{U}$ & Intact & $12 \mathrm{~mm}$ & RED/plates fixation & $2 \mathrm{yr}$ \\
\hline 8 & $\mathrm{U}$ & Intact & $11 \mathrm{~mm}$ & RED & $2 \mathrm{yr}$ \\
\hline 10 & B & Intact & $12 \mathrm{~mm}$ & RED/plate fixation & $3 \mathrm{yr}$ \\
\hline 11 & B & Intact & $13 \mathrm{~mm}$ & RED/bone graft & $1 \mathrm{yr}$ \\
\hline 12 & B & Intact & $13 \mathrm{~mm}$ & RED & $1 \mathrm{yr}$ \\
\hline 13 & $\mathrm{~B}$ & Missing & $4 \mathrm{~mm}$ & Le Fort I/bone graft & $5 \mathrm{yr}$ \\
\hline 14 & B & Intact & $10 \mathrm{~mm}$ & Internal distractors & $5 \mathrm{yr}$ \\
\hline
\end{tabular}

U: unilateral; B: bilateral; RED: rigid external distractor; yr: years. 


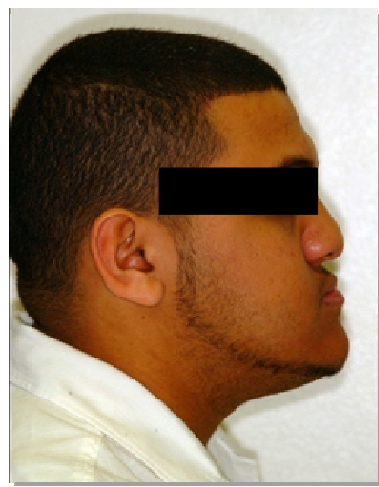

(a)

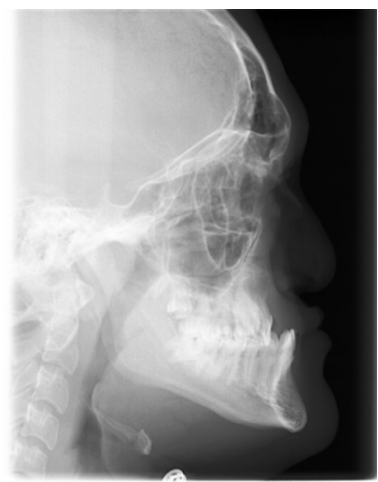

(b)

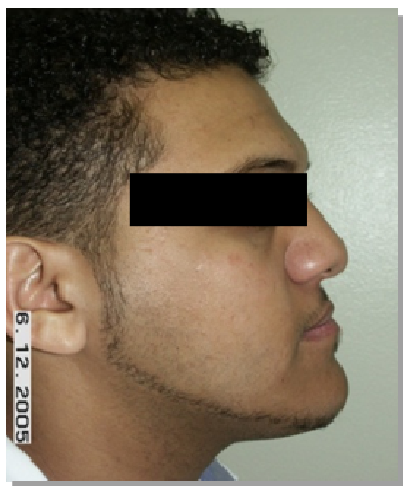

(c)

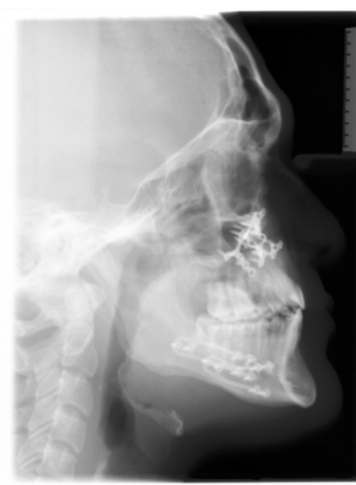

(d)

Figure 1. (a) Clinical lateral view of patient with unilateral cleft lip and palate; (b) Lateral cephalometric radiograph showing maxillary hypoplasia. Mandible underwent anterior and superior autorotation with overclosure of the vertical dimension with loss of facial height (pseudoprognathism); (c) Postoperative clinical picture showing lateral view; (d) Postoperative lateral cephalometric radiograph after le Fort I advancement and BSSO to seat occlusion.

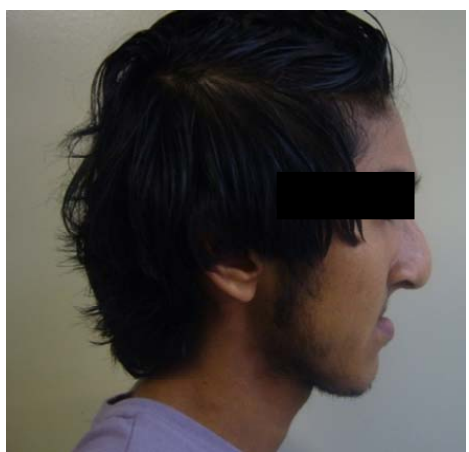

(a)

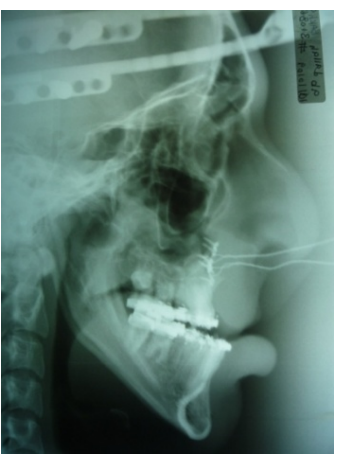

(b)

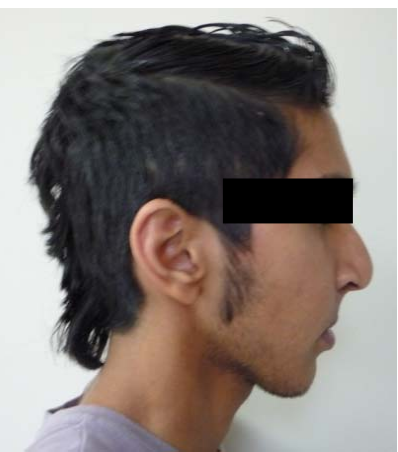

(c)

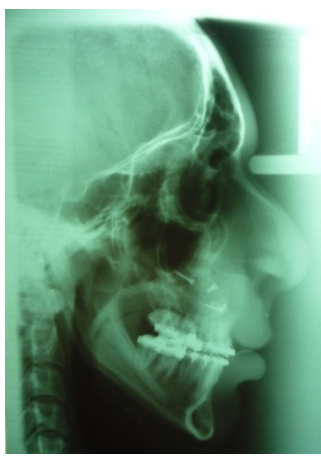

(d)

Figure 2. (a) Clinical picture showing lateral view with midface hypoplasia; (b) Immediate postoperative lateral cephalometric radiograph showing RED device in place before starting distraction osteogenesis; (c) Postoperative clinical picture showing lateral view after completing le fort I midface distraction osteogenesis; (d) Postdistraction osteogenesis lateral cephalometric radiograph.

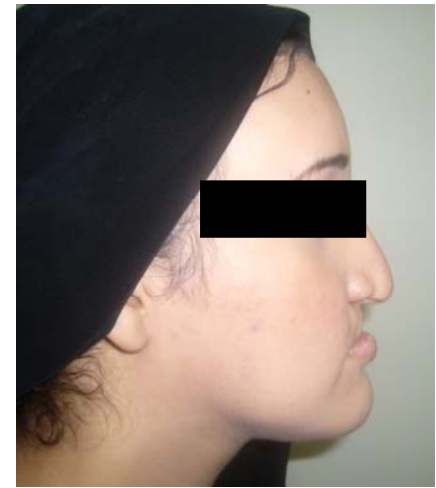

(a)

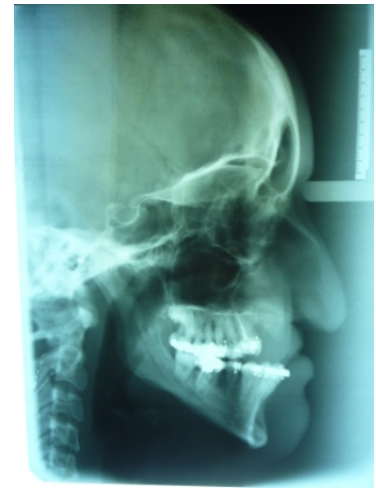

(b)

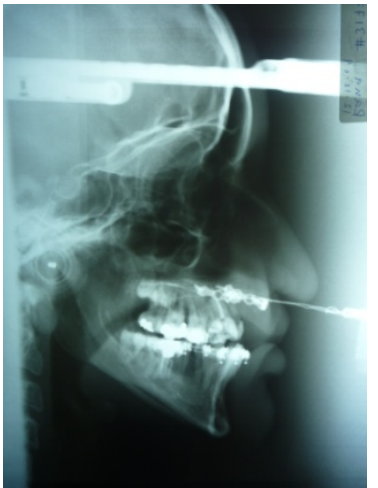

(c)

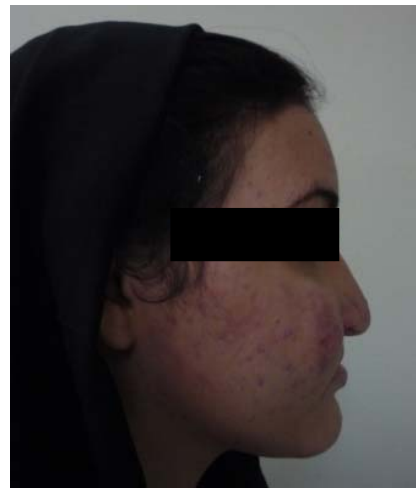

(d)

Figure 3. (a) Clinical picture showing lateral view with maxillary and midface hypoplasia; (b) Preoperative lateral cephalometric radiograph showing maxillary hypoplasia and missing premaxilla; (c) Lateral cephalometric radiograph showing maxillary advancement using RED; (d) Clinical picture showing lateral view after completing maxillary advancement using RED.

adjusting the distraction vector in the anterior maxillary region. Three patients underwent bone graft with screw fixation during the removal of the distractor due to the presence of a large bony defect in the anterior maxillary region. Two patients had a fibrous union and had to undergo plate fixation during distractor removal. All pa- 
tients showed dramatic improvement in facial esthetics and occlusion during the follow up period with no signs of relapse.

\section{DISCUSSION}

Cleft lip and palate patients normally undergo surgical soft tissue repair of the cleft lip and palate during infancy. The advantages of this surgical procedure shine brightly with the esthetic and functional improvement in the early days of the infant's life. Unfortunately, this pleasing effect is lost when impaired maxillary growth begins to make an appearance as the child grows. The resulting secondary deformities of the jaw and malocclusion are only a consequence of early soft tissue repair of the cleft palate .It has been reported that $25 \%$ to $60 \%$ of cleft lip and palate patients need to undergo maxillary advancement to correct the resulting midface hypoplasia $[5,6]$. Ross et al. [7] showed that about $25 \%$ of patients with unilateral cleft lip and palate develop maxillary hypoplasia that does not respond to orthodontic treatment alone.

Moreover, as a result of severe maxillary hypoplasia, the mandible often undergoes anterior and superior autorotation with subsequent over closure of the vertical dimension with a subsequent loss of facial height, pseudoprognathism, and upward inclination of the occlusal plane [8]. Maxillary advancement in cleft lip and palate patients can be achieved using conventional le Fort I osteotomy and plate fixation or using distraction osteogenesis (DO). Each technique has its indications and advantages.

Maxillary advancement using traditional one stage le Fort I osteotomy is an accepted treatment modality in treating maxillary deficiencies in cleft patients. It has the advantage of performing a single surgical procedure to advance the maxilla with surgical repair of residual oroantral fistula. However, higher relapse tendency is the major disadvantage. Hochban [9] noted a significantly higher relapse tendency in cleft patients who underwent maxillary le Fort I advancement (20\% - 25\%) compared to non cleft patients.

Many factors contribute to this high rate of relapse these include scarring from previous surgical repair of cleft lip and palate. The soft and hard tissue deficiencies are also contributing factors along with large maxillary advancements $[5,10]$. The amount of advancement is a major factor to consider before performing conventional le fort I advancement. It has been noted that large maxillary advancements will lead to a greater amount of relapse, however there are conflicting reports regarding the limit of maxillary advancement using conventional le Fort I in cleft patients. Some reports note that in large advancements are those exceeding $10 \mathrm{~mm}$ [11] .Others define large maxillary advancements as those beyond 8 $\mathrm{mm}$ [12] while others have reduced the limit of maxillary advancement to $5 \mathrm{~mm}$ with consideration of the palatal scar tissue formation $[13,14]$.

In most cases an interpositional bone grafts have been used to augment and stabilize the advanced maxilla, however the disadvantages include donor site morbidity and risk of resorption or infection. Many articles have proposed some modifications when applying le Fort I advancement in clefts in order to reduce the relapse tendency. They include proper freeing of the soft tissue and scar surrounding the maxilla in order to achieve adequate mobilization with special attention to the posterior and posterolateral aspects of the maxilla. In addition, the splint controlled position of the maxilla is over corrected by about 2 - $3 \mathrm{~mm}[11,15]$.We have applied these precautions when treating patients with conventional le Fort I.

Relapse of conventional le Fort I in cleft patients can still occur despite the application of a proper surgical technique and even with advancements that are not so large. This is not the case with Maxillary DO .This lead to the application of DO to advance the hypoplastic maxilla. Cleft patients with severe maxillary hypoplasia treated with DO show highly promising results [10]. It has numerous advantages over conventional le Fort I advancement. It allows large advancement of the underlying skeletal foundation with bony regeneration and elongation of the investing soft tissue. This gives better stability especially in cleft patients who require large advancements and present with severe palatal scaring [12]. Cheung compared relapse in clefts undergoing le fort I advancement of $5.3 \mathrm{~mm}$ with distraction group of $>6.7$. He reported better skeletal stability in the distraction group and greater relapse in the le Fort I advancemnt due to soft tissue stretch [16].

Relapse in large maxillary advancements with DO compared to smaller conventional le Fort I advancement generally show lower relapse during the follow up period. In addition, newly formed bone noted in the pterygoid region after maxillary distraction reduces the risk of relapse and spares the patient the need to undergo bone grafting. However, it is important to note that this newly formed bony trabeculae could only be seen 6 weeks after the active distraction phase, so a long consolidation period is of great importance to achieve stable results and preventing relapse [17]. We applied a strict consolidation period of three months in order to ensure and preserve the bony formation in the pterygoid region.

The soft tissue changes associated with maxillary DO should be considered .The soft tissue drape covering the lower third of the face dictates the limits of the underlying skeletal advancement. A sudden instant advancement of the maxilla using conventional le fort I does not give the same aesthetic soft tissue results that DO offers. This is due to the persistent soft tissue deficiency and the 
sudden stretch [18]. Therefore, more appealing soft tissue changes are obtained with DO. The nasal structure in clefts shows marked retrusion with retroclined con- figuration [18]. With le Fort I advancement nasal movement occurs at a 1:3 ratio. However with distraction the ratio is $1: 2$. DO produces positive soft tissue changes by increasing nasal projection and normalization of the nasolabial angle long with an increase in lip prominence. The concave facial profile becomes more convex [19]. The surgical technique can be modified by leaving the anterior nasal spine intact and high level le Fort I osteotomy may be required to increase nasal projection [20]. All of our patients underwent a high le Fort I osteotomy with preservation of the nasal spine except for the patients with a deficient premaxilla.

Although DO has several advantages over orthognathic surgery, its disadvantages and complications have been occasionally reported. DO requires a long duration to complete long with some difficulty to control the vector to achieve the favourable occlusion. The inability to guarantee formation of new bone in the osteotomy site is an issue of great importance. Nonunion has been reported in the literature and many contributing factors have been noted including age, mobile premaxilla and tooth loss [21]. Some surgical factors that lead to malunion of maxillary osteotomies include violation of the vascular pedicles, fracture of bony segments and poor stabilization [22]. These factors must be considered when performing maxillary osteotomies but with DO some additional factors may contribute to malunion they include patient compliance, short consolidation time, age, bilateral CLP, large DO advancement ( $>15 \mathrm{~mm})$, and compromised bone healing [23]. Two of our patients presented with malunion. One was a non compliant patient who tried repeatedly to remove the distractor by herself. The other patient underwent a large maxillary advancement $>20 \mathrm{~mm}$ and had very thin maxillary bone. They were both treated with bone grafting and plating during distractor removal. The creation of an anterior open bite and an increased mandibular plane angle was another complication we faced during the distraction phase. This was corrected by adjusting the vector of distraction followed by gradual closure of the open bite.

\section{CONCLUSION}

We have reported our protocol in managing adult cleft lip and palate patients with maxillary hypoplasia using traditional le Fort I and DO. Both techniques gave promising results providing having followed a good selection criteria; Patients with a severe maxillary hypoplasia of $6 \mathrm{~mm}$ or more and excessive palatal scaring are better treated with DO. Conventional le Fort I is better reserved for patients with less severe maxillary hypoplasia of less than $6 \mathrm{~mm}$ and less severe palatal scaring. It would be valuable to assess the associated soft tissue changes for all patients during the follow up phase.

\section{REFERENCES}

[1] Thongdee, P. and Samman, N. (2005) Stability of maxillary surgical movement in unilateral cleft lip and palate with preceding alveolar bone grafting. Cleft Palate-Craniofacial Journal, 42, 664-674. doi:10.1597/04-042R.1

[2] McCarthy, J., Schreiber, J., Karp, N., et al. (1992) Lenthening the human mandible by gradual distraction. Plastic and Reconstructive Surgery, 89, 1-10.

[3] Polley, J. and Figueroa, A. (1997) Management of severe maxillary deficiency in childhood and adolescence through distraction osteogenesis with an external adjustable rigid distraction device. Journal of Craniofacial Surgery, 8, 181-185. doi:10.1097/00001665-199705000-00008

[4] Rachmiel, A., Aizebud, D., Ardekian, L., et al., (1999) Surgically-assisted orthopaedic protraction of the maxilla in cleft lip and palate patients. International Journal of Oral and Maxillofacial Surgery, 28, 9-14. doi:10.1016/S0901-5027(99)80668-7

[5] Rachmiel, A., (2007) Treatment of maxillary cleft palate: Distraction osteogenesis versus orthognathic surgeryPart one: Maxillary distraction. Journal of Oral and Maxillofacial Surgery, 65, 753-757. doi:10.1016/j.joms.2006.08.010

[6] Panula, K., Lorius, B. and Pospisil, O. (1993) The need for orthognathic surgery in patients born with complete cleft palate or complete unilateral cleft lip and palate. Oral Surgery, Oral Diagnosis, 4, 23.

[7] Ross, R. (1987) Treatment variables affecting facial growth in complete unilateral cleft lip and palate: An overview of treatment and facial growth. Cleft PalateCraniofacial Journal, 24, 71-77.

[8] Scolozzi, P. (2008) Distraction Osteogenesis in the management of severe maxillary hypoplasia in cleft lip and palate patients. Journal of Craniofacial Surgery, 19, 1199-1214. doi:10.1097/SCS.0b013e318184365d

[9] Hochban, W., Ganss, C. and Austermann, K.H. (1993) Long-term results after maxillary-Advancement in patients with clefts. Cleft Palate-Craniofacial Journal, 30, 237-243. doi:10.1597/1545-1569(1993)030<0237:LTRAMA>2.3. $\mathrm{CO} ; 2$

[10] Figueroa, A., Polley, J., Friede, H., et al. (2004) Longterm skeletal stability after maxillary advancement with distraction osteogenesis using a rigid external distraction device in cleft maxillary deformities. Plastic and Reconstructive Surgery, 114, 1382-1392.

doi:10.1097/01.PRS.0000138593.89303.1B

[11] Precious, D. (2007) Treatment of retruded maxilla in cleft lip and palate-Orthognathic surgery versus distraction osteogenesis: The case for orthognathic surgery. Journal of Oral and Maxillofacial Surgery, 65, 758-761. doi:10.1016/j.joms.2006.08.011 
[12] Kanno, T., Mitsugi, M., Hosoe, M., Sukegawa, S., et al. (2008) Long-term skeletal stability after maxillary advancement with distraction osteogenesis in non-growing patients. Journal of Oral and Maxillofacial Surgery, 66, 1833-1846. doi:10.1016/j.joms.2007.10.013

[13] Wang, X., Yi, B., et al. (2005) Internal midface distraction in correction of severe maxillary hypoplasia seconddary to cleft lip and palate. Plastic and Reconstructive Surgery, 116, 51-60. doi:10.1097/01.PRS.0000169691.22783.29

[14] Cho, B. and Kyung, H. (2006) Distraction osteogenesis of the hypoplastic midface using a rigid external distraction system: The results of a one to six-year follow-up. Plastic and Reconstructive Surgery, 118, 1201-1212. doi:10.1097/01.prs.0000243563.43421.0b

[15] Haers, P., Ge, Z., Locher, M., et al. (1997) Stability of maxillary osteotomies in cleft patients approached by minimal incisions and stabilised by plate osteosynthesis and splint without IMF. In: Lee, S.T., Ed., Transactions of the 8th International Congress on Cleft Palate and Related Craniofacial Anomalies, Stamford Press, Singapore City, 630-634.

[16] Cheung, L., Chua, H. and Hagg, M. (2006) Cleft maxillary distraction versus orthognathic surgery: Clinical morbidities and surgical relapse. Plastic and Reconstructive Surgery, 118, 996-1008. doi:10.1097/01.prs.0000232358.31321.ea

[17] Kusnoto, B., Figueroa, A. and Polley, J. (2001) Radiographic evaluation of bone formation in the pterygoid region after maxillary distraction with a rigid external distraction (RED) device. Journal of Craniofacial Surgery,
12, 109-117. doi:10.1097/00001665-200103000-00003

[18] Drew, S. (2000) Maxillary advancement with distraction osteogenesis by use of a rigid external distraction device: A 1-year follow-up (Discussion). Journal of Oral and Maxillofacial Surgery, 58, 969. doi:10.1053/joms.2000.8798

[19] Ko, E., Figueroa, A. and Polley, J. (2000) Soft tissue profile changes after maxillary advancement with distraction osteogenesis by use of a rigid external distraction device: A 1-year follow-up. Journal of Oral and Maxillofacial Surgery, 58, 959. doi:10.1053/joms.2000.8735

[20] Freihofer, H. (1977) Change in nasal profile after maxillary advancement advancement in cleft and non-cleft patients. Journal of Maxillofacial Surgery, 5, 20. doi:10.1016/S0301-0503(77)80071-4

[21] Krimmel, M., Cornelius, C., Gulicher, D., et al. (2005) Longitudinal cephalometric analysis after maxillary distraction osteogenesis. Journal of Craniofacial Surgery, 16, 683-688. doi:10.1097/01.scs.0000168779.39969.c1

[22] Van Sickels, J. and Tucker, M. (1990) Management of delayed union and nonunion of maxillary osteotomies. Journal of Oral and Maxillofacial Surgery, 48, 10391044. doi:10.1016/0278-2391(90)90285-A

[23] He, D., Genecov, D. and Barcelo, R. (2010) Nonunion of the external maxillary distraction in cleft lip and palate: analysis of possible reasons. Journal of Oral and Maxillofacial Surgery, 68, 2402-2411. doi:10.1016/j.joms.2009.09.018 